\title{
Atomistic Molecular Modeling of the Effect of Chromophore Concentration on the Electro-optic Coefficient in Nonlinear Optical Polymers
}

\author{
M. R. Leahy-Hoppa,* P. D. Cunningham, J. A. French, and L. M. Hayden \\ Department of Physics, University of Maryland, Baltimore County, Baltimore, Maryland 21250
}

Received: November 11, 2005; In Final Form: March 2, 2006

\begin{abstract}
We employ fully atomistic molecular modeling to investigate the concentration dependence of the electrooptic coefficient of two guest-host polymer composites. Using classical molecular dynamics, we record the time-evolution of the guest-host system under the application of an external electric field. Through analysis of the orientation of the nonlinear optical chromophores in the guest-host composite with respect to the direction of the external electric field, we calculate the orientational parameter $N<\cos ^{3} \theta>$, with $N$ being the number density of chromophores in the composite. This parameter is directly proportional to the electrooptic coefficient. We find agreement between the concentration dependence of the electro-optic coefficient calculated through our simulation and that from experimental data and also from Monte Carlo models.
\end{abstract}

\section{Introduction}

Nonlinear optical (NLO) polymers are used in a wide variety of applications from electro-optic (EO) modulators ${ }^{1,2}$ to optical data storage. ${ }^{3}$ The EO coefficient of NLO polymers plays an important role in the characterization and utilization of these material systems. The EO coefficient is essentially a measure of the quality of the material for specific applications. Therefore, for the continued development of these types of materials, it is necessary to understand the behavior of the EO coefficient and be able to predict whether a proposed new system is an enhancement over existing materials.

Theoretical development of the acentric ordering of the NLO chromophores has been an area of focus for Prezhdo and coworkers. ${ }^{4-7}$ Additionally, the characterization of the EO coefficient of NLO polymers has been investigated experimentally ${ }^{8-10}$ as well as through Monte Carlo statistical mechanical modeling. ${ }^{11-13}$ Specifically, the dependence of the EO coefficient on the NLO chromophore concentration in the material has been investigated both experimentally and through Monte Carlo simulations. ${ }^{11-13}$ Robinson et al. ${ }^{11}$ point out that the EO coefficient in NLO polymer systems exhibits both shape and concentration dependences. The mean-field theory investigations by Prezhdo and Dalton ${ }^{4-7}$ have also shown the shape and concentration dependence of the polar ordering of the chromophores in the NLO systens. For systems with spherically shaped chromophores, the EO coefficient exhibits a linear increase with concentration at low to moderate concentrations but exhibits a sublinear increase at high concentrations. All other parameters being equal, elliptically shaped chromophores in NLO polymer systems exhibit a similar type of behavior; however, the sublinear response of the EO coefficient occurs at a more moderate concentration of chromophores in the system with high concentrations of chromophores resulting in a reduction of the EO coefficient past a peak in the EO activity.

Although this phenomenon, which we call the roll-off of the electro-optic coefficient, has been shown both experimentally and theoretically through Monte Carlo simulations, it has not been observed (until now) at the atomistic level of theory. The

* Corresponding author e-mail: megan.leahy.hoppa@umbc.edu.
Monte Carlo simulations performed by Robinson et al. ${ }^{11}$ do not yield atomistic structural information regarding the molecular systems since those simulations use dipoles with given shapes on a periodic lattice. Atomistic molecular modeling provides the opportunity to investigate these materials in a more detailed fashion, providing structural information and feedback to the chemists and material scientists designing the new molecules.

Several groups have previously investigated the EO coefficient in these types of NLO polymer materials through electric field poling simulations. ${ }^{11,14,15}$ Since the EO coefficient is directly proportional to the orientational order of the chromophores in the materials, the poling order provides a direct way of investigating the EO coefficient of a variety of materials. Kim and Hayden ${ }^{14}$ investigated a dilute system of an NLO chromophore with a small dipole moment $(\sim 4 \mathrm{D})$ in the polymer poly(methyl methacrylate) (PMMA) finding agreement between the achieved polar order and that predicted by a Boltzmann distribution in the noninteracting rigid gas model. MakowskaJanusik et al. ${ }^{15}$ have also investigated dilute guest-host polymer systems using atomistic molecular modeling with several different chromophores. Although there have been several studies simulating NLO polymer poling processes, none have yet exhibited the experimentally observed concentration dependence of the poling order of NLO polymer systems using an atomistic level of theory.

In this work, we have built upon the investigations of Kim and Hayden, ${ }^{14}$ and investigate systems containing chromophores with large dipole moments, equivalent to those used experimentally. In this article we first present the methodology used in performing our simulations as well as the levels of theory available to describe the behavior of the poled systems. We then discuss the results from several series of simulations highlighting similarities and differences between our atomistic simulations, experimental data, and previous Monte Carlo simulations.

\section{Methods}

We perform fully atomistic molecular modeling using classical force field methods. Classical force field methods employ 
Newtonian mechanics to observe the time-evolution of a system. We use the Consistent Valence Force Field (CVFF) 16,17 potential to determine the forces and therefore the subsequent velocities of the atoms during our simulations. The definition of the potential energy of the system can be expressed as

$$
V=V_{\text {bond }}+V_{\text {angle }}+V_{\text {torsion }}+V_{\text {oop }}+V_{\text {nonbond }}+\left(V_{\text {field }}\right)
$$

The $V_{\text {field }}$ term is used for the application of the external electric field and is only included during the poling stage of the simulations and is expressed as $V_{\text {field }}=\sum_{i} q_{\mathrm{i}} \mathbf{E} \cdot \mathbf{r}_{i}$. Both the $V_{\text {bond }}$ and the $V_{\text {angle }}$ terms are described using quadratic potentials, $V_{\text {bond }}=\sum_{b} H_{b}\left(b-b_{\mathrm{o}}\right)^{2}$ and $V_{\text {angle }}=\sum_{\theta} H_{\theta}\left(\theta-\theta_{\mathrm{o}}\right)^{2}$. The torsional potential is expressed as $V_{\text {torsion }}=\sum_{\varphi} H_{\varphi}(1+s \cos (n \varphi))$ and the out-of-plane interaction as $V_{\text {oop }}=\sum_{\chi} H_{\chi} \chi^{2}$. The nonbonded interactions are described in terms of two potentials, a van der Waals potential, $V_{\mathrm{vdW}}=\sum \epsilon\left[\left(\left(r^{*} / r\right)^{12}-2\left(r^{*} / r\right)^{6}\right)\right]$, and a Coulomb potential, $V_{\text {Coul }}=\sum\left(q_{i} q_{j} / \epsilon r_{i j}\right)$. The parameters $H, s, n$, and $r^{*}$ are set in the force field. The parameter $\epsilon$, the dielectric constant, is user defined. To calculate the potential energy due to the external electric field, we use the summation of the dot product of the coordinate of each atom and the force exerted on each atom by the external electric field

$$
V_{\text {field }}=\sum_{i=1}^{n} \mathbf{r}_{i} \cdot\left(q_{i} \mathbf{E}\right)
$$

where $E$ is the vector applied field, the $r_{i}$ are the coordinate vectors of each of the atoms, and the $q_{i}$ are the corresponding charges for each atom at the position $r_{i}$.

The ordering of these types of systems can be described using several levels of theory. The noninteracting rigid gas model uses the third-order Langevin function to predict the orientational ordering of the chromophores as a function of the poling parameter $p=\mu \mathrm{E} / k T$

$$
L_{3}(p)=\left(1+6 / p^{2}\right) L_{1}(p)-2 / p=\left\langle\cos ^{3}(\theta)\right\rangle
$$

with

$$
L_{1}(p)=\operatorname{coth}(p)-1 / p=\langle\cos \theta\rangle
$$

This model seems to be adequate to describe relatively dilute concentrations of chromophores in these systems. ${ }^{14,15}$ This model, however, does not include intermolecular electrostatic interactions. Robinson and Dalton ${ }^{11}$ have used the methodology of London ${ }^{18}$ to predict the orientational ordering expected with the inclusion of intermolecular interactions. Making use of an "effective field" felt by the dipoles from the applied poling field, and other dipole fields, they have expressed the expected ordering in these systems to be

$$
\left\langle\cos ^{3} \theta\right\rangle=L_{3}(p)\left[1-L_{1}^{2}(W / k T)\right]
$$

with

$$
W=\left(1 / R^{6}\right)\left[\left(2 \mu^{4} / 3 k T\right)+2 \mu^{2} \alpha+3 I \alpha^{2} / 4\right]
$$

The average interchromophore distance, $R$, molecular polarization, $\alpha$, and ionization potential, $I$, are taken into account in the chromophore-chromophore interaction energy, $W$.

We investigated two guest-host polymeric systems consisting of a PMMA polymer host and two chromophore guests, dinitrovinylmethylpyridine, DNVMP, and EZ-FTC (Figure 1). Previous work in our group has shown ${ }^{14}$ that PMMA itself does

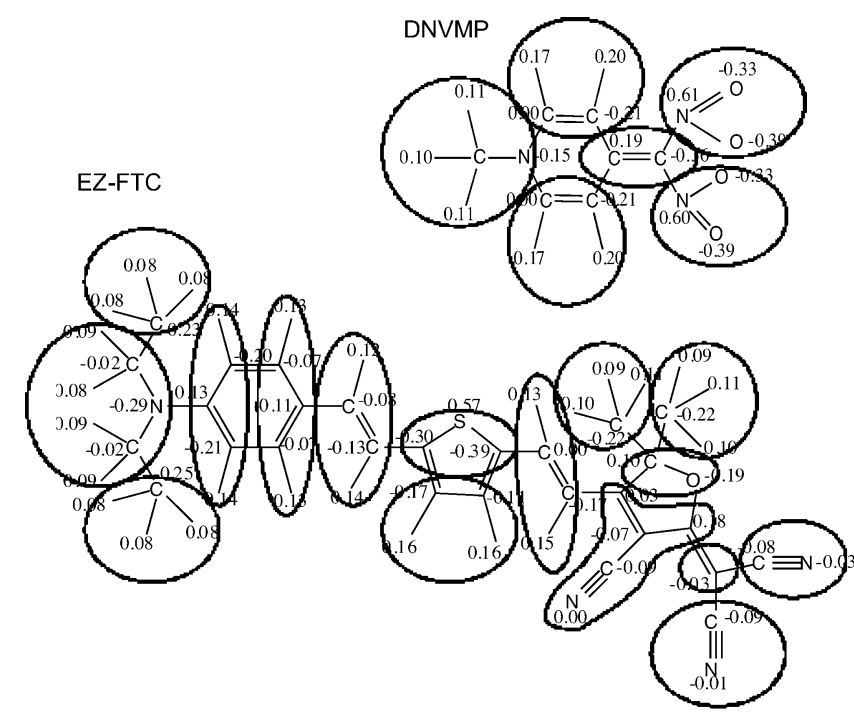

Figure 1. Chemical structures of the chromophores DNVMP and EZFTC. MOPAC charges are listed next to the atoms. Circles indicate the charge groups. DNVMP has a dipole moment of $12.5 \mathrm{D}$, and EZFTC has a dipole moment of $14 \mathrm{D}$ using MOPAC assigned partial charges on CVFF force field geometries.

not significantly reorient under the application of the external electric field.

The systems are prepared with a predetermined concentration of chromophores by weight with respect to the polymer. We use a Monte Carlo amorphous cell generation method ${ }^{19}$ for the creation of three-dimensional amorphous systems. We initially create one system at a given density, $0.98 \mathrm{~g} / \mathrm{cm}^{3}$, and perform a constant pressure (NPT) study to determine the glass transition temperature ${ }^{20}$ of the simulated system. Subsequent systems for the poling studies use parameters dictated by the glass transition temperature $\left(T_{\mathrm{g}}\right)$ study simulations. We employ the CVFF ${ }^{16,17}$ force field in each step of our simulations. Our simulations are performed with Cerius $2^{21}$ software from Accelrys. Molecular dynamics (MD) and energy minimizations occur within Cerius2 with the Discover (98.0) ${ }^{21}$ program. The force field parameters used in this study have previously been reported. ${ }^{14}$

All the MD simulations are performed using the velocity Verlet $^{22}$ method with $1 \mathrm{fs}$ time steps. The temperature of the system is controlled using a velocity scaling method. ${ }^{23}$ We use group-based cutoffs for nonbonded interaction distances. The group-based cutoffs for the nonbonded interactions are $9.5 \AA$ for the van der Waals term and are equal to half the size of the cubic simulation box for the Coulomb interactions. Group-based nonbond cutoffs are used to avoid artificial splitting of dipoles. Groups are assigned (Figure 1) to have no more than 5 heavy atoms and no more than 8 atoms total and to be charge neutral within $0.3 \mathrm{e}\left(4.80 \times 10^{-20} \mathrm{C}\right)$. Since partial charges assigned by the CVFF force field under-represent the dipole moment of the chromophore, partial charges on the individual atoms within the chromophores are assigned using MOPAC ${ }^{24}$ charges from a geometry optimization simulations. For example, the EZ-FTC chromophore has a dipole moment of $14 \mathrm{D}$ calculated using the MOPAC partial charges on the molecule with the CVFF force field geometries, yet the CVFF force field assigned charges in the force field geometry result in a dipole moment of about 5 D. Since the MOPAC partial charges more closely represent the experimental dipole moment of the chromophores, we assign the MOPAC partial charges to all the atoms in the chromophores in the force field geometries.

For the $T_{\mathrm{g}}$ study, we first create the cell at a given density and concentration by weight of the chromophore and perform 


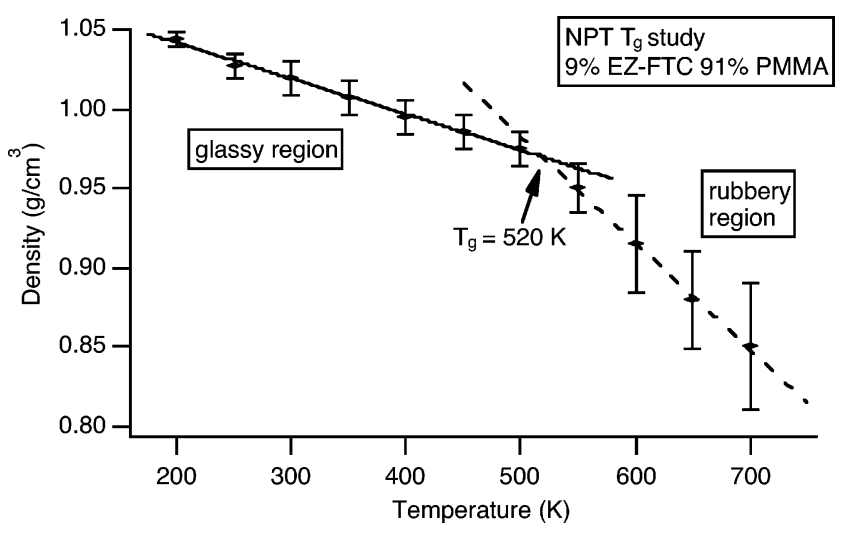

Figure 2. EZ-FTC/PMMA $T_{\mathrm{g}}$ study. The dashed line is a linear fit to the rubbery region data, and the solid line is a linear fit to the glassy region data. The point where the two fit lines cross defines the simulation glass transition temperature, $T_{\mathrm{g}}$.

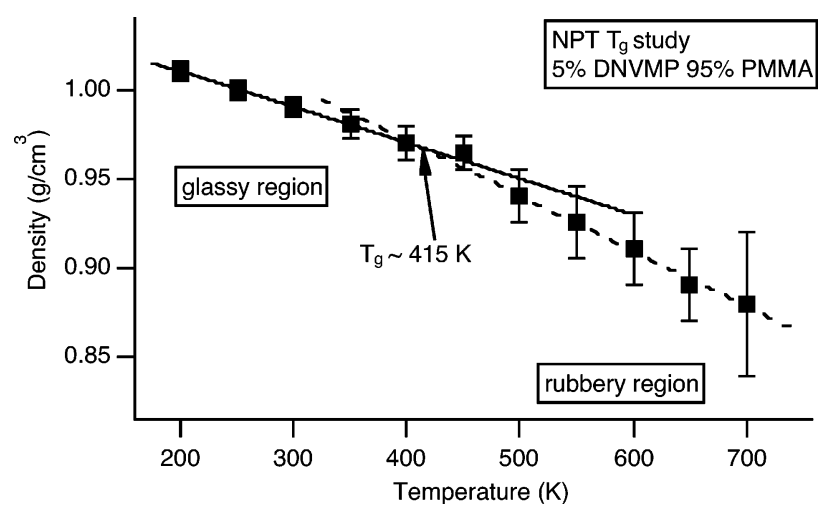

Figure 3. DNVMP/PMMA $T_{\mathrm{g}}$ study. The dashed line is a linear fit to the rubbery region, and the solid line is a linear fit to the glassy region. The crossing point of the two fit lines defines the simulation $T_{\mathrm{g}}$ of the system.

an energy minimization on the cell. The energy of the cell is minimized using the conjugate gradient method with the convergence criteria of $0.1 \mathrm{kcal} / \mathrm{mol} / \AA$. We perform constant pressure-temperature (NPT) molecular dynamics (MD) at 700 $\mathrm{K}$ and atmospheric pressure to equilibrate the system at the starting temperature for the simulations. The equilibration

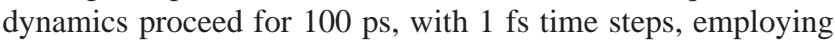
the Berendsen pressure control method ${ }^{25}$ in every NPT MD step. We use group-based cutoffs in order to avoid artificial splitting of dipoles for both van der Waals and Coulomb nonbonded interactions. The lowest potential energy configuration of the NPT MD is minimized for 500 steps with the conjugate gradient method using the same convergence criteria as the first minimization step. We performed 300 ps of NPT MD to determine the equilibrium density of the system at each temperature from $700 \mathrm{~K}$ to $200 \mathrm{~K}$ in $50 \mathrm{~K}$ increments. The final configuration of the MD at each temperature is used as the initial configuration for the next lower temperature MD simulation. At each temperature, we record the equilibrium density of the system (Figures 2 and 3). There are two identifiable regions on the density versus temperature graphs, the glassy and rubbery regions. The breaking point between the two slopes in the graph identifying these regions points to the simulation glass transition temperature. The simulated $T_{\mathrm{g}}$ is higher than the experimental $T_{\mathrm{g}}$ because of the extremely high cooling rate $\left(1.7 \times 10^{11} \mathrm{deg} /\right.$ s). In both systems, we also determined, through simulations not shown here, that increased concentration of the chromophore in the system has a plasticizing effect, thereby decreasing the simulation $T_{\mathrm{g}}$ of the system. Therefore, using the parameters

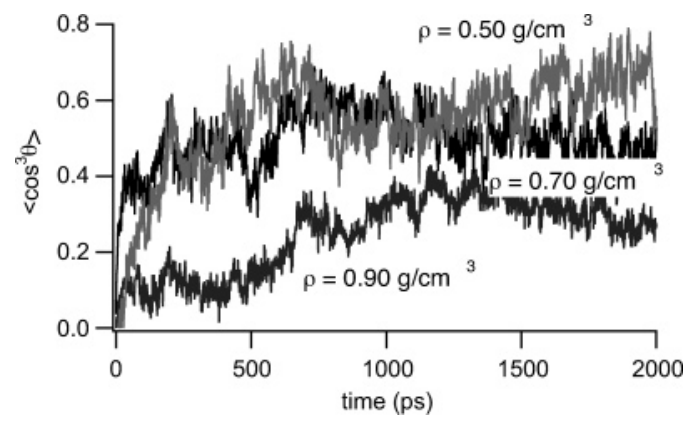

Figure 4. Poling order as a function of system density for the EZFTC/PMMA system at $9 \%$ concentration by weight.

set by the lowest concentration system, we are assured all simulations occur in the rubbery region of the material.

Our initial investigation into the EZ-FTC system at low concentration and at a density of $0.9 \mathrm{~g} / \mathrm{cm}^{3}$, corresponding to that which is determined by the glass transition temperature study, yielded a poling order of the system which is less than that predicted by the noninteracting rigid gas model. Under the same conditions, all the other guest-host systems we investigated, in this work and previous work, exhibited ordering predicted by the noninteracting rigid gas model. The EZ-FTC chromophore, however, is much larger in size than any other chromophore we had previously studied. The EZ-FTC chromophore is more than double the length of the DNVMP chromophore. We attribute the reduced degree of polar order in the EZ-FTC/PMMA system to steric hindrance due to the large size of the chromophore. To overcome this problem, we reduced the density of the system until the ordering predicted by the noninteracting rigid gas model, $L_{3}(\mathrm{p})=0.60$ with $p=$ 5.65, was matched by the MD simulations (Figure 4). Previous investigations have shown ${ }^{14,15}$ that at high poling fields and low concentration, the polar order of these systems is the same as that predicted by the noninteracting rigid gas model.

Once the system parameters have been determined, through the NPT $T_{\mathrm{g}}$ studies and the poling efficiency study, we then perform the poling study. First we create $30-100$ configurations and minimize their energies using the conjugate gradient method with the convergence criteria of $0.1 \mathrm{kcal} / \mathrm{mol} / \AA$. The configurations with the lowest energies are chosen. The equilibration procedure includes constant volume-temperature (NVT) molecular dynamics (MD) followed by 500 steps of energy minimization on the lowest potential energy configuration of the NVT-MD. The final configuration of the second minimization is the initial configuration for the poling MD. This procedure ensures that the systems are not in an energetically unstable configuration where consecutive steps of MD could experience a large change in energy which would cause the simulation to prematurely end. Following the equilibration of the systems, we enter the poling and analysis stages of the investigation. The poling stage of the MD consists of $2 \mathrm{~ns}$ of NVT MD recording the configuration once every 1 ps. During the analysis, we calculate the angle the dipole moment vector of each chromophore makes with the applied electric field vector. We compare our results both with experimental results and with previous Monte Carlo simulations of the poling of NLO molecules performed by Robinson and co-workers. ${ }^{11,12}$ The simulations were performed on a Xeon $2 \mathrm{GHz}$ based LINUX server.

\section{Results}

We have examined two guest-host systems containing the same polymer host, PMMA, with differing chromophores, 


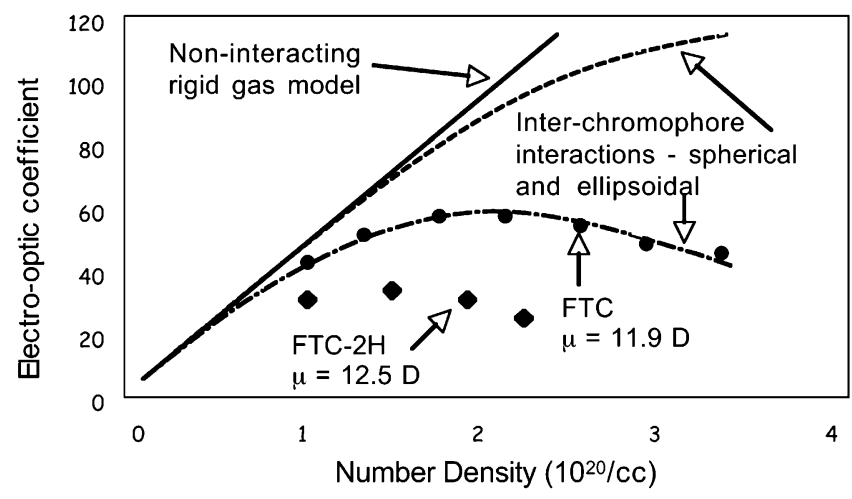

Figure 5. Behavior of the EO coefficient as a function of number density or concentration. The circles and diamonds are experimental data for two variants of FTC-based chromophores. The lines represent statistical mechanical levels of theory. The straight line corresponds to the noninteracting point dipole model. The curved dashed line corresponds to a full consideration of intermolecular interactions with chromophores treated as spheres. The solid curved line represents full consideration of intermolecular interactions with chromophores treated as nonpenetrating ellipsoids. (Adapted from Figure 2 [ref 11]).

DNVMP and EZ-FTC (see Figure 1). The difference in size and shape of the chromophores allow us to investigate the dependencies of the EO coefficient on the shape and size of the chromophore while minimal changes occur in the dipole moment. The DNVMP chromophore is significantly shorter in length than the EZ-FTC chromophore, $8 \AA$ compared with 20 $\AA$. This difference in lengths of the chromophores also contributes to the difference in shapes of the chromophores. The DNVMP chromophore is much less elliptical in shape than the EZ-FTC chromophore.

Previous investigations of the shape dependence of the EO coefficient by Robinson and Dalton ${ }^{11}$ using Monte Carlo and statistical mechanical methods have illustrated the difference between the concentration dependent EO coefficient of elliptical and spherical chromophores (see Figure 5). Prezhdo and Dalton ${ }^{6}$ have additionally investigated the shape dependence of the chromophores through mean-field theory calculations. Although the noninteracting rigid gas model predicts a linear increase of the EO coefficient with increased chromophore concentration, the shapes of the chromophores alter the concentration effects of the EO coefficient. At high concentrations, spherical chromophores experience a more gentle roll-off of the EO coefficient as compared to elliptical chromophores. The points on the graph in Figure 5 illustrate the experimentally observed concentration dependence of the EO coefficient for two different elliptically shaped chromophores.

The results of our atomistic investigation of the DNVMP/ PMMA system can be seen in Figures 6 and 7. We show the concentration dependence of the EO coefficient through the product of the orientational factor, $<\cos ^{3} \theta>$, and the number density of chromophores. In Figure 6, the circles correspond to typical experimental conditions, where $p \approx 1$. The squares on the graph correspond to poling conditions with an applied poling field six times greater than typical experimental poling conditions. With this high poling field, the system experiences a linear increase in the EO coefficient equivalent to that predicted by the noninteracting rigid gas model, $L_{3}(\mathrm{p})=0.64$. At the highest concentration, the polar order is slightly less than that predicted by the point dipole model (solid line). Figure 7 shows an expanded view of the $p=1$ simulations. The decrease of the EO coefficient at high concentrations can easily be observed and is similar to that shown by both Robinson and Dalton ${ }^{11}$ (Figure 5) for the spherical chromophores and by Prezhdo and

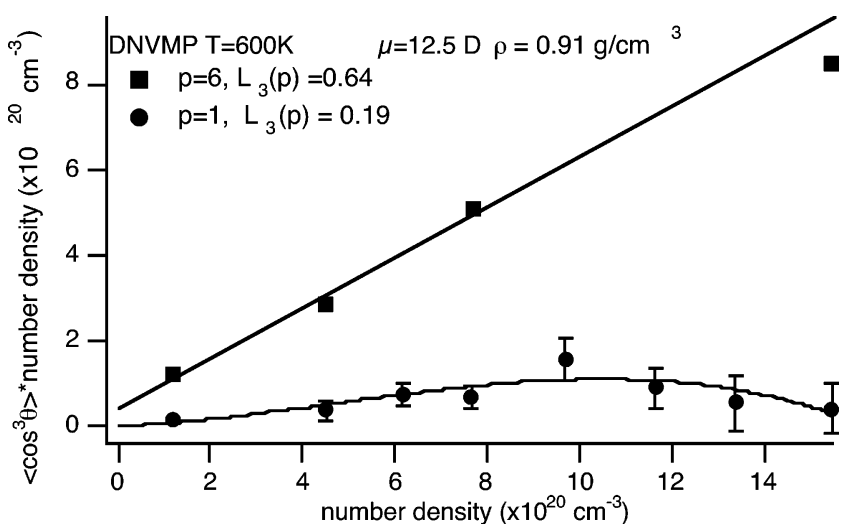

Figure 6. EO activity $\left(<\cos ^{3} \theta>*\right.$ number density $\left.\propto r_{33}\right)$ as a function of chromophore concentration for two different poling fields for the DNVMP/PMMA system. The straight line is the noninteracting rigid gas model prediction of the EO activity. The curved line through the circles is a guide to the eye.

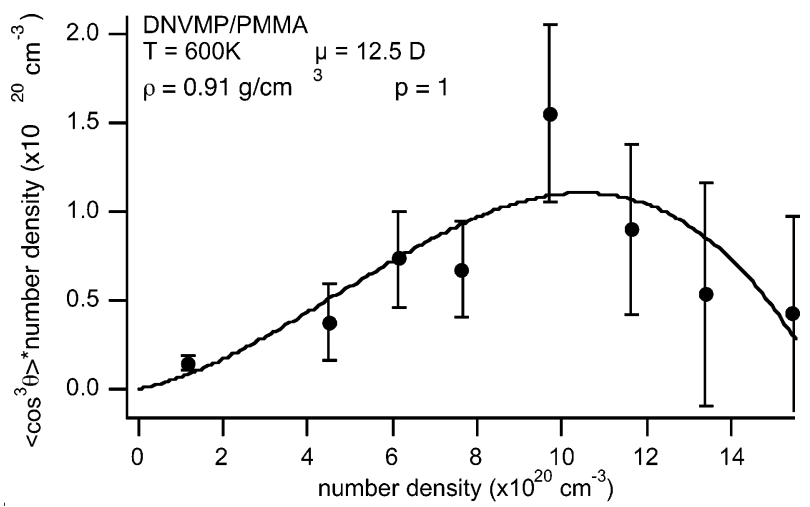

Figure 7. Roll-off of the EO activity $\left(<\cos ^{3} \theta>*\right.$ number density $\propto$ $\left.r_{33}\right)$ for the DNVMP/PMMA system. The line is a guide to the eye.

Dalton. ${ }^{6}$ Although Robinson and Dalton do not carry out the calculation to high enough concentrations to observe the decrease in EO coefficient for spherical chromophores, the trend indicates the peak EO activity occurs at higher concentrations than for the elliptical chromophores.

Our investigation of the EZ-FTC/PMMA system can also be compared with Robinson and Dalton's Monte Carlo simulations. ${ }^{11}$ We performed the poling simulations at three different poling field strengths. Figure 8 shows the results of the three poling investigations as well as a comparison with Robinson and Dalton's data. In Figure 8, the circles correspond to typical experimental poling conditions, $p=1$. The diamonds and squares have $p=2.8$ and 5.7. Under the highest poling field, the systems exhibit orientational ordering predicted by the noninteracting rigid gas model, $L_{3}(\mathrm{p})=0.6$. This is to be expected since the poling field dominates all other fields, such as dipole-dipole and induced-dipole interactions. The linear trend in the EO coefficient predicted by the noninteracting rigid gas model can easily be observed. Decreasing the poling field, the chromophores can begin to interact, and we observe the concentration dependent decrease in the EO coefficient with increased concentration for elliptical chromophores shown by Robinson and Dalton in Figure 5. The inset graph in Figure 8 shows Robinson and Dalton's Monte Carlo simulation data for an elliptical chromophore with a similar dipole moment to the EZ-FTC chromophore, under similar simulation conditions as our case of $p=2.8$. The trends in our EZ-FTC data show a slight shift in the peak EO activity to lower concentrations in comparison with the Monte Carlo simulation; however, it has been shown by Robinson and co-workers ${ }^{11,12}$ that the location 


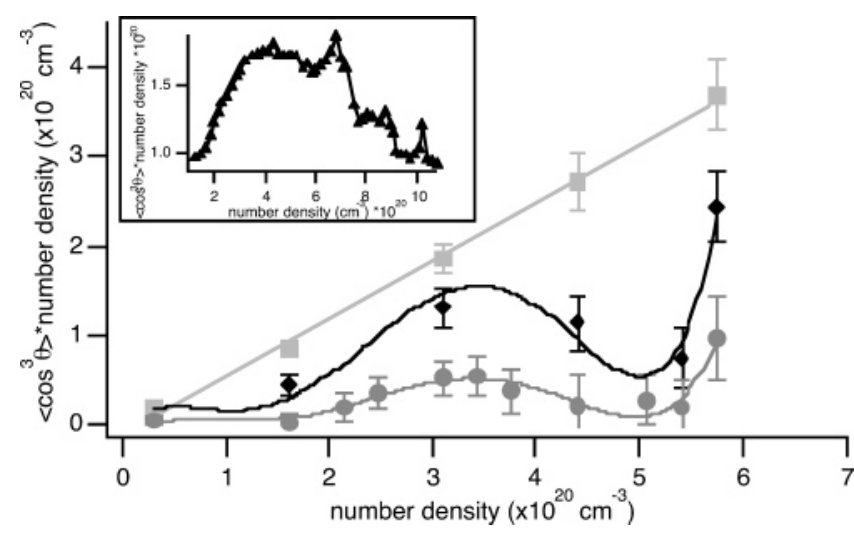

Figure 8. Concentration dependence of the EO activity $\left(<\cos ^{3} \theta>*\right.$ number density $\propto r_{33}$ ) of the EZ-FTC/PMMA system for three poling field strengths. Field strengths varied from $0.18 \mathrm{kV} / \mu \mathrm{m}(p=1)$ to $1 \mathrm{kV} / \mu \mathrm{m}$ $(p=5.7)$. At the two lower field strengths, the roll-off of the EO activity can be seen at a concentration of $3.5 \times 10^{20}$ molecules $/ \mathrm{cm}^{3}$. This peak EO activity occurs at a similar concentration and with a similar strength to that shown by Robinson and Dalton in their MC simulations. ${ }^{11} \mathrm{At}$ the highest concentration, $5.8 \times 10^{20}$ molecules $/ \mathrm{cm}^{3}$, the systems exhibit phase separation of the chromophore molecules and the polymer chain as well as aggregation of the chromophores into liquid-crystalline smectic-C-type ordering (Figure 9). The straight line is the noninteracting rigid gas model. The curved lines are guides to the eye. Insert: (Adapted from Figure 9 [ref. 11]). Monte Carlo simulations of EO activity as a function of number density. of the peak of the EO activity is highly dependent on the dipole moment of the chromophore. The EZ-FTC chromophore has a slightly larger dipole moment, $14 \mathrm{D}$, in comparison with the dipole moment of Robinson's chromophore, 13 D. Robinson has shown ${ }^{11}$ that increasing the dipole moment of a chromophore yields a shift to lower concentrations of the peak in the EO activity.

The data in Figure 8 show an additional increase in the EO activity after $5 \times 10^{20} \mathrm{~cm}^{-3}$. Visual inspection of the systems showed smectic-C type liquid-crystalline ordering. An example snapshot can be seen in Figure 9. The balls and sticks are the chromophores, whereas the thick sticks on the right-hand side of the picture represent the polymer chain. At the highest concentrations, where the largest ordering occurs in our simulations, the systems are approximately $85-90 \%$ chromophore (by weight). In these cases, we observe ordered layering of the chromophores within the majority of the systems. The positive $z$-direction is the direction of the external electric field applied during the simulations. Experimentally, the largest realizable chromophore concentration using this type of chromophore is near $25 \%$ by weight. Higher chromophore concentrations result in chromophore aggregation and crystallization. The liquidcrystalline-like ordering of the chromophores seen in the Figure 9 occurred in a simulation with $89 \%$ chromophore concentration by weight and is therefore not observable experimentally.

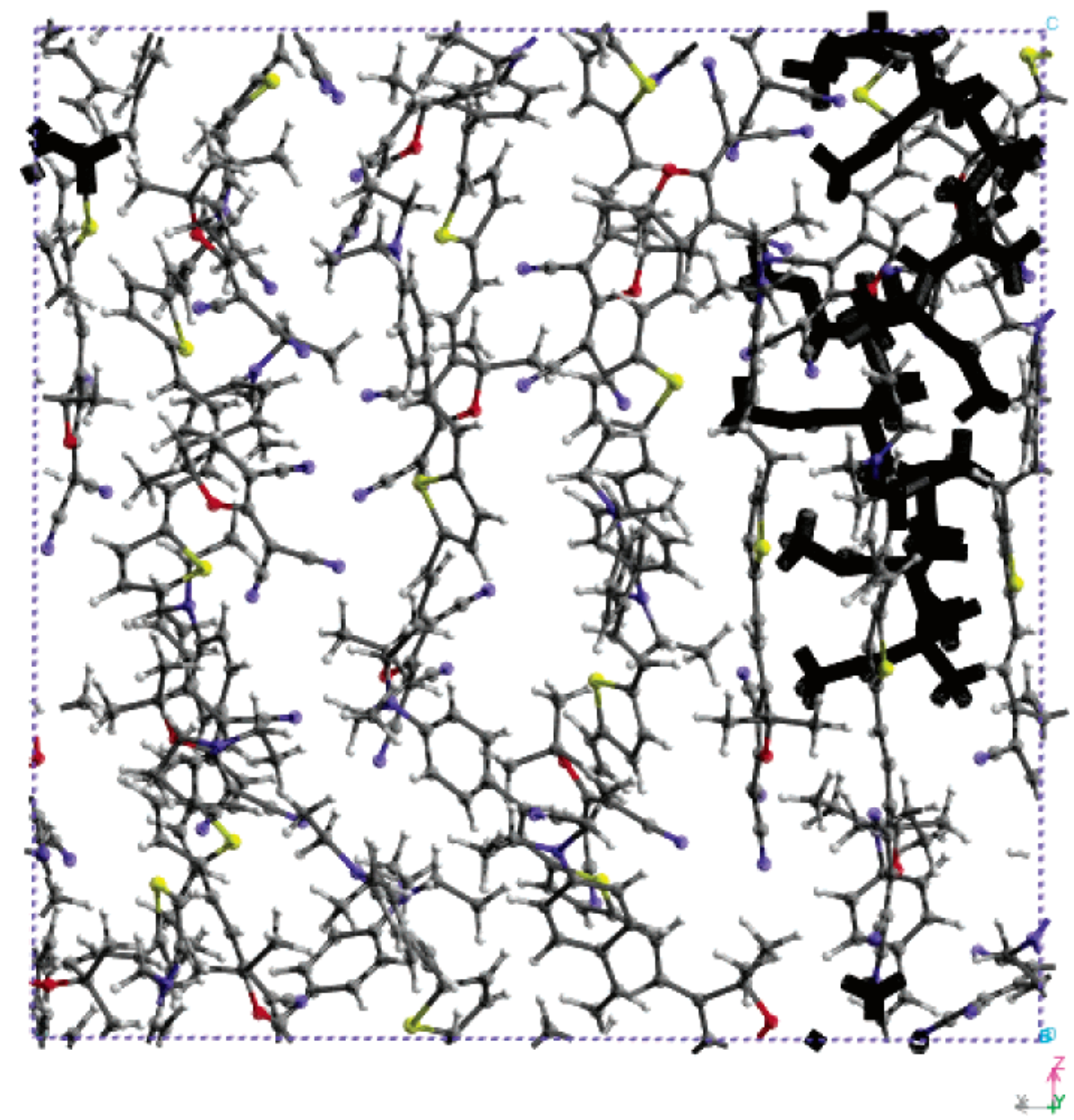

Figure 9. Snapshot of the EZ-FTC/PMMA system at the highest concentration $\left(5.8 \times 10^{20}\right.$ molecules $/ \mathrm{cm}^{3}$ or $89 \%$ chromophore by weight). The polymer is represented by the thick black cylinders and can be seen mostly on the right-hand side in the middle to upper portion of the cell. The balls and sticks are the chromophore molecules. The grouping of the chromophores can be seen throughout the cell. Six different layers of groups of chromophores can be seen in this picture. The positive $z$-direction is the direction of the poling field in this simulation. Note the coordinate system at the lower right-hand corner of the figure. 
Several levels of theory have been developed to describe the behavior of these types of molecular systems. The noninteracting rigid gas model can be applied in cases of dilute systems ${ }^{14,15}$ as well as in cases of extremely large applied electric fields. Under typical experimental conditions, however, the noninteracting rigid gas model is inadequate to describe the behavior of most systems. Robinson and Dalton ${ }^{11}$ have developed a level of theory which includes electrostatic intermolecular interactions and predicts many of the features observed in experiments. The Monte Carlo simulations of Robinson and Dalton consider the chromophores as dipoles on a periodic lattice with a given shape. Prezhdo and co-workers ${ }^{4-7}$ have additionally provided meanfield theory calculations investigating both shape and concentration effects in polymeric systems. Our atomistic simulations include polymer molecules with the chromophores and also allow the molecules to move freely within the unit cell. Further work is needed to merge the best part of each type of simulation. Monte Carlo models are fast and efficient, whereas atomistic models provide detailed information about atom positions. A coarse-grained intermediate approach may prove to be the most successful.

\section{Discussion}

We employed fully atomistic molecular modeling to investigate the concentration dependence of the electric field poling behavior of two nonlinear optical chromophores in a guesthost polymer configuration. We compared our results with both experimental observations and Monte Carlo simulations. Using atomistic molecular modeling techniques, we have demonstrated the ability to adequately model the poling behavior of these types of polymeric systems as a function of NLO chromophore concentration.

We have developed a systematic method for poling guesthost polymer systems using atomistic molecular modeling. This method involves careful characterization of the molecular system in order to ensure ordering of the chromophores under conditions similar to that used in typical experiments. The density and poling temperature are found using constant pressure, temperature (NPT) dynamics and allow for the determination of the appropriate poling field strength. The strength of the applied poling field is an important parameter to accurately determine due to the delicate balance between the strengths of the poling field interaction with the chromophores and that of the intermolecular electrostatic interactions. Too large of an applied poling field will overwhelm all intermolecular interactions therefore dominating the behavior of the EO coefficient with increased chromophore concentration.

This method allows for the characterization and poling of guest-host systems in such a way that systematic comparison between guest-host systems can be performed in order to determine relative strengths of polar order in the systems. The comparison between different NLO chromophores in the guesthost systems allows us to determine whether a new chromophore shows improved EO activity over existing materials. This information allows us to guide the development of improved materials.
Our fully atomistic demonstration of the concentration dependence concurs with both experimentally observed phenomena and that observed through Monte Carlo simulations. Although the MC simulations are invaluable to the field of research, limited structural information can be obtained due to the number of approximations used within the simulations. Our simulations, accounting for all the atoms in the system, allow more detailed structural investigations of the systems and the individual molecules within the system. Having now established the ability to perform the same type of simulations as the MC simulations at a more detailed level of theory, we can now begin to examine new molecular architectures such as dendrimers and oblate spheroidally shaped chromophores.

Acknowledgment. The authors thank Drs. Bruce Robinson, Bruce Eichinger, and Larry Dalton for helpful discussions. Research support is gratefully acknowledged from the NSF Center on Materials and Devices for Information Technology Research (CMDITR), DMR-0120967.

\section{References and Notes}

(1) Shi, Y.; Zhang, C.; Zhang, H.; Bechtel, J. H.; Dalton, L. R.; Robinson, B. H.; Steier, W. H. Science 2000, 288, 119.

(2) Zhang, C.; Dalton, L. R.; Oh, M. C.; Zhang, H.; Steier, W. H. Chem. Mater. 2001, 13, 3043.

(3) Rahn, M. D.; West, D. P.; Khand, K.; Shakos, J. D.; Shelby, R. M. Appl. Opt. 2001, 40, 3395.

(4) Pereverzev, Y. V.; Prezhdo, O. V.; Dalton, L. R. Chem. Phys. Lett. 2003, 373, 207.

(5) Pereverzev, Y. V.; Prezhdo, O. V. Phys. Rev. E 2000, 62, 8324.

(6) Pereverzev, Y. V.; Prezhdo, O. V.; Dalton, L. R. Phys. Rev. B 2002, 65, 0521041.

(7) Pereverzev, Y. V.; Prezhdo, O. V.; Dalton, L. R. Chem. Phys. Lett. 2001, 340, 328 .

(8) Luo, J.; Haller, M.; Ma, H.; Liu, S.; Kim, T.-D.; Tian, Y.; Chen, B.; Jang, S.-H.; Dalton, L. R.; Jen, A. K.-Y. J. Phys. Chem. B 2004, 108 , 8523 .

(9) Sandalphon; Kippelen, B.; Meerholz, K.; Peyghambarian, N. Appl. Opt. 1996, 35, 2346.

(10) Han, S. H.; Wu, J. W. J. Opt. Soc. Am. B 1997, 14, 1131.

(11) Robinson, B. H.; Dalton, L. R. J. Phys. Chem. A 2000, 104, 4785.

(12) Nielsen, R. D.; Rommel, H. L.; Robinson, B. H. J. Phys. Chem. B 2004, 108, 8659 .

(13) Dalton, L. R.; Harper, A. W.; Robinson, B. H. Proc. Natl. Acad. Sci. U.S.A. 1997, 94, 4842.

(14) Kim, W.-K.; Hayden, L. M. J. Chem. Phys. 1999, 111, 5212

(15) Makowska-Janusik, M.; Reis, H.; Papadopoulos, M. G.; Economou, I. G.; Zacharopoulos, N. J. Phys. Chem. B 2004, 108, 588.

(16) Hagler, A. T.; Huler, E.; Lifson, S. J. Am. Chem. Soc. 1974, 96, 5319 .

(17) Kitson, D. H.; Hagler, A. T. Biochemistry 1988, 27, 5246.

(18) London, F. Trans. Faraday Soc. 1937, 33, 8.

(19) Amorphous Builder; Molecular Simulation Inc.: San Diego, CA.

(20) Soldera, A. Macromol. Symp. 1998, 133, 21.

(21) Cerius2; Molecular Simulation Inc.: San Diego, CA.

(22) Swope, W. C.; Anderson, H. C.; Berens, P. H.; Wilson, K. R. J. Chem. Phys. 1982, 76, 637.

(23) Woodcock, L. V. Chem. Phys. Lett. 1971, 10, 257.

(24) Stewart, J. J. P. MOPAC, a semiempirical molecular orbital program; 6.0 ed.; Quantum Chemical Program Exchange No. 455, 1983.

(25) Berendsen, H. J. C.; Postma, J. P. M.; van Gunsteren, W. F.; DiNicola, A.; Haak, J. R. J. Chem. Phys. 1984, 81, 3684. 